\title{
A STREAMLINE APPROACH FOR HISTORY-MATCHING PRODUCTION DATA
}

\author{
SUPRI TR - 125
}

\author{
By \\ Yuandong Wang and Anthony R. Kovscek
}

\section{TOPICAL REPORT}

\author{
For the period ending \\ June 2001 \\ Work Performed Under Contract No. DE-FC26-00BC15311 \\ Prepared for \\ U.S. Department of Energy \\ Assistant Secretary for Fossil Energy \\ Thomas Reid, Project Manager \\ National Petroleum Technology Office \\ P.O. Box 3628 \\ Tulsa, OK 74101
}




\section{DISCLAIMER}

This report was prepared as an account of work sponsored by an agency of the United States Government. Neither the United States Government nor any agency thereof, nor any of their employees, makes any warranty, express or implied, or assumes any legal liability or responsibility for the accuracy, completeness, or usefulness of any information, apparatus, product, or process disclosed, or represents that its use would not infringe privately owned rights. Reference herein to any specific commercial product, process, or service by trade name, trademark, manufacturer, or otherwise does not necessarily constitute or imply its endorsement, recommendation, or favoring by the United States Government or any agency thereof. The views and opinions of authors expressed herein do not necessarily state or reflect those of the United States Government or any agency thereof. 


\section{TABLE OF CONTENTS}

$\underline{\text { Page }}$

List of Figures

iv

Acknowledgements

iv

Abstract

$\mathrm{V}$

1. Introduction

1

1.1 Previous Work 2

2. Method 4

3. Match of Fractional Flow Curve 6

$3.1 \quad$ Simplifying the Inverse System 9

4. Match of Flow Rate and Pressure 11

5. Permeability Updates 11

6. Steps of the Approach 12

7. Example Applications 12

7.1 Large Scale Trends, Cases 1 and $3 \quad 13$

$\begin{array}{lll}7.2 & \text { Effect of Mobility Ratio, Case } 3 & 14\end{array}$

7.3 Variable Injection Rate, Case 4 16

8. Discussion 16

$\begin{array}{lr}\text { 9. Conclusions } & 20\end{array}$

$\begin{array}{ll}\text { 10. Nomenclature } & 20\end{array}$

$\begin{array}{ll}\text { 11. References } & 21\end{array}$ 


\section{LIST OF FIGURES}

$\underline{\text { Page }}$

1. Case 1, comparison of permeability fields 24

2. Case 1, fractional flow curves 25

3. Case 1, error reduction 26

4. Case 2, comparison of permeability fields 27

5. Case 2, fractional flow curves 28

6. Case 2, error reduction $\quad 29$

7. Case 3, comparison of permeability fields 30

8. Case 3, (a) superposition of tracer slug injection solution 31

(b) concentration match

9. Case 3, error reduction 32

10. Case 3, fractional flow curves 33

11. Case 4, comparison of permeability fields 34

12. Case 4, fractional flow curves 35

13. Case 4, pressure history 36

14. Case 4, error reduction 37

\section{ACKNOWLEDGEMENTS}

This paper was prepared with the support of the U.S. Department of Energy, under Award No. DE-FC26-00BC15311. However, any opinions, findings, conclusions, or recommendations expressed herein are those of the authors and do not necessarily reflect the views of DOE. Additionally, the support of the SUPRI-A Industrial Affiliates is gratefully acknowledged. 


\begin{abstract}
This study proposes and develops a streamline approach for inferring field-scale effective permeability distributions based on dynamic production data including producer water-cut curve, well pressures, and rates. The streamline-based inverse approach simplifies the history-matching process significantly.

The basic idea is to relate the water-cut curve at a producer to the water breakthrough of individual streamlines. By adjusting the effective permeability along streamlines, the breakthrough time of each streamline is found that reproduces the reference producer fractionalflow curve. Then the permeability modification along each streamline is mapped onto cells of the simulation grid. Modifying effective permeability at the streamline level greatly reduces the size of the inverse problem compared to modifications at the grid-block level. The approach outlined here is relatively direct and rapid. Limitations include that the forward flow problem must be solvable with streamlines, streamline locations do not evolve radically during displacement, no new wells are included, and relatively noise-free production data is available. It works well for reservoirs where heterogeneity determines flow patterns. Example cases illustrate computational efficiency, generality, and robustness of the proposed procedure. Advantages and limitations of this work, and the scope of future study, are also discussed.
\end{abstract}




\section{INTRODUCTION}

History-matching plays an important role in monitoring the progress of displacement processes and predicting future reservoir performance. Historical production data is routinely collected and it carries much information, although convoluted, that is useful for reservoir characterization and description of reservoir heterogeneity (Vasco and Datta-Gupta, 1997; Grinestaff, 1999). In this paper, the concept of streamlines is applied to develop an automatic method for inferring the permeability distribution of a reservoir based on the history of pressure, flow rate, and water cut at producers.

The properties of streamlines are used in deriving the inverse method, and so a brief review of streamline methodology follows. Streamline and streamtube techniques are approximate reservoir simulation methods proposed some years ago (Higgins and Leighton, 1962; Higgins et al., 1964) that have undergone recent intense study (Hewett and Behrens, 1991; Thiele et al., 1996; Peddibhotla et al., 1997). They are most accurate when heterogeneity determines flow paths and the recovery process is dominated by displacement (viscous forces) as opposed to gravity or capillarity. (Blunt et al., 1996). A streamline is tangent everywhere to the instantaneous fluid velocity field and, for a symmetric permeability tensor, streamlines are perpendicular to isobars or iso-potential lines. Streamlines bound streamtubes that carry fixed volumetric flux, and in some cases, flow rate is assigned to streamlines (Thiele et al., 1996; Batycky et al., 1997). For this reason, we use the terms streamline and streamtube interchangeably.

The streamline method assumes that displacement along any streamline follows a onedimensional solution, and there is no communication among streamlines. Thus, the flow problem is decomposed into a set of one-dimensional flow simulations linked by common boundary conditions. A streamline must start and end at a source to maintain continuity. In a streamlinebased approach, pressure equations are solved independent of saturation equations. The decoupling of pressure equations from saturation equations speeds up significantly the simulation by reducing the number of times that the pressure field must be updated and greatly reduces the number of equations to solve.

For unit mobility ratio and constant boundary conditions, the streamline distribution remains unchanged throughout the displacement process. Therefore, the pressure field or streamline distribution only needs to be solved once and saturation solutions can be mapped 
along streamlines. For non-unit mobility ratio, there are two common approaches to treat streamlines. One is to fix the streamline geometry and allow the flow rate to change during the displacement process. (Hewett and Yamada, 1997; Portella and Hewett, 1997). The other is to update the streamline distribution and distribute the flow rate equally among the streamtubes (Thiele et al., 1995a; 1995b). In the second case, both the pressure field and streamline geometry must be updated periodically.

For complete descriptions of the various streamline formulations and inclusive reviews of the history of streamlines for predicting reservoir flow, please refer to references (Hewett and Behrens, 1991; Blunt et al., 1996; Hewett and Yamada, 1997; King and Datta-Gupta, 1998).

In this study, the streamline simulator 3DSL by Batycky et al. (1997) is employed for forward simulation. In short, after solving the pressure field and the streamline distribution, 3DSL assigns equal flow rate to each streamline. Then a one-dimensional saturation solution, either analytical or numerical, is solved along the streamlines. Periodically, the streamline saturation distribution is mapped onto the multidimensional grid, the pressure equation is resolved, and streamline geometry redetermined. There is no detectable reduction in accuracy with this technique for incompressible and viscous determined flows. (Wang et al., 1999).

\subsection{Previous Work}

Most approaches to history-matching field data manipulate permeability at the grid-block level, and hence, demand a great amount of computational work because there are many gridblocks in a typical simulation. Integration of production data with reservoir description remains an important issue because of the prevalence of production data and the information that it carries about the reservoir. In this brief review, we focus on work that is most similar to our method to follow. Other approaches to history matching are based upon simulated annealing ${ }^{18}$, sensitivity coefficients (Wen et al., 1998; Vasco, et al., 1998; Chu et al., 1995), and parameter estimation approaches (Landa and Horne, 1997).

Sensitivity coefficient techniques compute the sensitivity of the objective function to the change of permeability of a cell or a set of cells and solve an inverse system that can be very large and somewhat difficult to construct (Chu et al., 1995; Landa and Horne, 1997). Sensitivity coefficient methods might also be computationally expensive if the sensitivity coefficients are evaluated numerically by running multiple simulations. Chu et al. (1995) developed a 
generalized pulse spectrum technique to estimate efficiently the sensitivity of wellbore pressure to gridblock permeability and porosity. Other work employed sensitivity coefficients in the integration of well test information, production history, and time-lapse seismic data (Landa and Horne, 1997).

Vasco et al. (1998) combined streamlines and a sensitivity coefficient approach while integrating dynamic production data. They employ streamlines to estimate sensitivity coefficients analytically thereby greatly speeding up the procedure. The streamline analysis allows them to "line up" the first arrival of injected fluid at production wells and then match the production history. This technique remains a grid-block-level optimization approach as all of the cells from the flow simulation are used to describe reservoir heterogeneity.

Sensitivity coefficients have also been employed in a scheme to identify the geometry of geological features such as faults and the dimensions of flow channels. (Rahon et al., 1998). In essence, the technique is to minimize an objective function incorporating single and/or twophase production data. The parameters for minimization are the size and interfacial area of geological bodies rather than grid-block parameters.

Simulated annealing, as applied by Gupta et al. (1994), perturbs permeability in a set of grid-blocks and evaluates energy objective functions or the degree of misfit between simulated and desired results. This process is stochastic and it is not guaranteed that a perturbation will decrease the energy level. The decision of whether or not to accept the perturbation is based on the change of energy caused by this perturbation. Perturbations that increase the degree of mismatch are accepted with a frequency that decreases with increase in the error. Many iterations are usually required to obtain an acceptable solution. In general, the computational costs of incorporating production data using simulated annealing become very large if a reservoir simulation must be conducted for each iteration.

Reservoir characterization approaches such as geostatistics do not explicitly account for field production data. It is a major task to determine the geostatistical realizations that are consistent with injection and production data. Generally, this involves conducting flow simulations for many different geostatistical simulation realizations. Efficient conditioning of permeability fields to both a geostatistical model and production data is discussed by $\mathrm{Wu}$ et al. (Wu et al., 1998). In other work, Wen et al. (1998a; Wen et al., 1998b) also present a geostatistical approach to the inverse problem of integrating well production data. They adapt the 
sequential self-calibration (SSC) inverse technique to single-phase, multi-well, transient pressure and production rate data. The SSC method is an iterative, geostatistically-based inverse method coupled with an optimization procedure that generates a series of coarse grid two-dimensional permeability realizations. In later work, they combine SSC with analytical computation of sensitivity coefficients using streamline distributions (Wen et al., 1998). The output realizations correctly reproduce the production data. In both instances, this approach is applied for singlephase flow.

An interesting question that arises with any match to production data is the accuracy of the match. Lepine et al. (1998) combine error analysis with a gradient-based technique to compute the uncertainty in estimates of future performance based upon history-matched models. Their method helps to identify and select the parameters of a given reservoir model that most sensitively affect the match.

The remainder of this paper presents the development of a streamline-based historymatching approach where streamline effective permeability is manipulated rather than grid-block level data. An algorithm for mapping inverse streamline information onto the grid is also discussed. Next, several synthetic cases are used to explore the proposed approach. Discussion and conclusions complete the paper.

\section{METHOD}

We propose a two-step method to match dynamic production data and infer reservoir heterogeneity. The first step is to modify the permeability distribution at the streamline level based on the difference between simulation results and field data for water cut, pressure drop, and flow rate. By matching the fractional-flow curve through manipulation of the permeability field, we try to capture reservoir heterogeneity. The second step is to map the streamline permeability modification onto the grid-blocks. Then flow simulation is performed to check the match. The above process is iterated until convergence.

A number of assumptions or simplifications were made in order to begin. Most originated from the streamline method used for forward flow displacement calculations. Permeability is assumed to be isotropic and the fluids are incompressible. Gravity and capillary forces are taken as negligible. Throughout we consider a single injector/producer pair with no changes in well configuration, no infill wells, no conversion of producers to injectors, or vice 
versa. Also, we assume that other parameters such as porosity, relative permeability, and boundary conditions are known accurately. Once the method has been established to work under these conditions, it will be extended.

Necessary output from the forward streamline simulator includes streamline coordinates and evenly distributed flow among streamlines. Of course, the flow problem must be solvable by the streamline simulator. Our method requires monotonic increasing fractional flow. Therefore, field production data for slug tracer injection must be processed by superposition before performing the inverse process. An example tracer case follows that illustrates the success of this superposition treatment. Likewise, noisy field production data must be filtered and pre-processed to obtain a good match.

The proposed inverse process makes use of streamline properties. In a multi-phase displacement, each streamline breakthrough contributes a small amount to the fractional-flow curve at a producer. Throughout, we use the terms water-cut and fractional flow interchangeably. Because of the equal flow rate property of streamlines in forward flow simulation with 3DSL, every streamline breakthrough contributes the same amount to the fractional flow. Therefore, by ordering the streamlines with respect to their breakthrough time, we discretize the fractional-flow curve and relate various segments to the breakthrough of individual streamlines. In this sense, our proposed method is similar to the work of Vasco et al. (1998); however, we do not compute sensitivity coefficients nor is our formulation of the inverse problem similar. When the fractional-flow curve of the forward simulation result for a given permeability does not match the field water-cut curve, we infer the streamlines responsible for the difference between the fractional flow curves. Then based on the relation between streamline breakthrough time and effective permeability, a modification of effective permeability along streamlines can be computed to match the production data. The objective function, as defined below, indicates the error of the simulation result compared to the field data:

$$
E=w_{t} E_{t, n}+w_{p} E_{p, n}+w_{q} E_{q, n}
$$

In Equation (1), $E_{t, n}, E_{p, n}$, and $E_{q, n}$ are errors in the dimensionless breakthrough time of individual streamlines, pressure, and flow rate at the producer, respectively. The terms $w_{t}, w_{p}$, 
$w_{q}$ are weights for dimensionless breakthrough time of individual streamlines, pressure, and flow rate at producer $i$, respectively. For simplicity, we weight each term identically.

We decouple the computation of streamline permeability modification to match flow rate and/or pressure from that to match fractional flow. To capture heterogeneity, we need to solve an inverse system to match the fractional flow curve. This is the most important part of this study and is therefore discussed in detail.

\section{MATCH OF FRACTIONAL FLOW CURVE}

The degree of mismatch between reference and history-matched results is computed as

$$
E_{t}=\frac{1}{N_{s l}} \sum_{i=1}^{N_{s l}}\left|E_{t_{B T}, i}\right|
$$

where $N_{s l}$ is the number of streamlines connected to the producer. The error $E_{t_{B T}, i}$ refers to breakthrough time of streamline $i$ as defined below

$$
E_{t_{B T}, i}=t_{D, B T, i}^{C}-t_{D, B T, i}^{R}
$$

where $t_{D, B T, i}^{C}$ and $t_{D, B T, i}^{R}$ are the computed, C, and reference, R, dimensionless breakthrough times (pore volume injected) for the $i^{\text {th }}$ streamline, respectively.

Permeability modification of the $i^{\text {th }}$ streamline alters the breakthrough time of not only the $i^{t h}$ streamline, but possibly the other streamlines. Therefore, all the streamlines must be considered, at least initially, and a system of equations has to be solved.

The system to solve is

$$
\left[\begin{array}{ccccc}
a_{11} & a_{12} & a_{13} & \cdots & a_{1 N} \\
a_{21} & a_{22} & a_{23} & \cdots & a_{2 N} \\
a_{31} & a_{32} & a_{33} & \cdots & a_{3 N} \\
\cdots & & & & \\
a_{N 1} & a_{N 2} & a_{N 3} & \cdots & a_{N N}
\end{array}\right]\left[\begin{array}{c}
\Delta k_{1} \\
\Delta k_{2} \\
\Delta k_{3} \\
\cdots \\
\Delta k_{N}
\end{array}\right]=-\left[\begin{array}{c}
E_{t, B T, 1} \\
E_{t, B T, 2} \\
E_{t, B T, 3} \\
\cdots \\
E_{t, B T, N}
\end{array}\right]
$$

where $E_{t, B T, i}$ is defined in Eq. (3), $\Delta k_{j}$ is the modification of effective permeability along streamline $j$ required to get a match, and $\boldsymbol{a}_{i j}$ is the sensitivity of breakthrough time 
(dimensionless) of the $\boldsymbol{i}^{\text {th }}$ streamline to the effective permeability of the $\boldsymbol{j}^{\text {th }}$ streamline. These derivatives are defined as

$$
a_{i j}=\frac{\partial t_{D, B T, i}}{\partial k_{j}}
$$

where $t_{D, B T, i}$ is the dimensionless breakthrough time of streamline $\boldsymbol{i}$, and $\boldsymbol{k}_{\boldsymbol{j}}$ is the effective permeability along streamline $\boldsymbol{j}$. Because streamlines are non-communicating, the derivatives can be approximated by applying Dykstra and Parsons (1950) method for non-communicating layers. The method relates the breakthrough time of different layers to the effective permeability of each layer. For unit mobility ratio and piston-like displacement, the approximation is exact. The generalization of Dykstra and Parsons method to streamlines is discussed elsewhere (Hewett and Yamada, 1997).

The breakthrough time for streamline $i$ is calculated as

$$
t_{D, B T, i}=\frac{\sum_{k=1}^{N_{s l}}(\bar{A} \bar{\phi} L)_{k} x_{D, k, i}}{\sum_{k=1}^{N_{s l}}(\bar{A} \bar{\phi} L)_{k}}
$$

where $\boldsymbol{L}$ is the length of a streamline, $x_{D, k, i}$ is the the fraction of pore volume of streamline $k$ swept when streamline $i$ breaks through. Equivalently, $x_{D, k, i}$ is the dimensionless time of flight of the displacing front ${ }^{[12]}$ along streamline $\mathrm{k}$ when the $i^{\text {th }}$ streamline breaks through. Obviously, $x_{D, i, i}=1$. For those streamlines that break through earlier than streamline $i, x_{D, k, i}$ can be greater than 1 . The symbols $\bar{\phi}_{k}$ and $\overline{A_{k}}$ represent the average porosity and average crosssectional area of streamline $k$ respectively. They are defined as

$$
\begin{aligned}
& \bar{A}=\int_{0}^{1} A\left(x_{D}\right) d x_{D} \\
& \bar{\phi}=\int_{0}^{1} \phi\left(x_{D}\right) d x_{D}
\end{aligned}
$$

Now, define the ratio of pore volume of streamline $k$ over the total pore volume as

$$
V_{D, k}=\frac{(\bar{A} \bar{\phi} L)_{k}}{\sum_{i=1}^{N_{s l}}(\bar{A} \bar{\phi} L)_{i}}=\frac{V_{P, k}}{V_{P, T}}
$$


where the subscript $D$ denotes dimensionless, $V_{P, k}$ is the pore volume of streamline $k$, and $V_{P, T}$ is the total pore volume. Equation (6) can be rewritten as

$$
t_{D, B T, i}=\sum_{k=1}^{N_{s l}} V_{D, k} x_{D, k, i}
$$

Then by applying the chain rule, Eq. (5) is evaluated:

$$
\frac{\partial t_{D, B T, i}}{\partial k_{j}}=\sum_{k=1}^{N_{s l}} \frac{\partial t_{D, B T, i}}{\partial x_{D, k, i}} \frac{\partial x_{D, k, i}}{\partial k_{j}}=\sum_{k=1}^{N_{s l}} V_{D, k} \frac{\partial x_{D, k, i}}{\partial k_{j}}
$$

Dykstra and Parsons (1950) method provides $x_{D, k, i}$ in terms of the effective permeability of all the streamlines; hence, the summation above must be computed for all $\mathrm{k}$ streamlines. The formula for calculating $x_{D, k, i}$ for unit mobility ratio is slightly different from that for non-unit mobility ratio.

For unit mobility ratio, the pressure field as well as the streamline distribution remains unchanged throughout the displacement process for constant boundary conditions. When breakthrough happens at streamline $i$, the front position at streamline $k$ is calculated by

$$
x_{D, k, i}=c_{i k} \frac{k_{k}}{k_{i}}
$$

where $c_{i k}$ is a constant related to the length of the streamline $i$ and $k$. It is computed from streamline geometry. Applying this definition and completing the partial derivative indicated in Eq. (7) yields

$$
\frac{\partial t_{D, i}}{\partial k_{j}}=\sum_{k=1}^{N_{s l}} V_{D, k} \frac{\partial x_{D, k, i}}{\partial k_{j}}= \begin{cases}-\frac{1}{k_{i}^{2}} \sum_{k=1, k \neq i}^{N_{s l}} c_{i k} V_{D, k} k_{k}, & \text { if } i=j \\ c_{i j} V_{D, j} / k_{i}, & \text { if } i \neq j\end{cases}
$$

This procedure can be repeated for non-unit mobility ratios given the standard Dykstra-Parsons result (Lake, 1989), where $x_{D, k, i}$ is a function of the permeability of streamlines $i$ and $k$, and the end-point mobility ratio.

For non-unit mobility ratio cases, we approximate streamlines as non-evolving in inverse calculations. This assumption works well if heterogeneity is a dominant factor during 
displacement. Therefore, it is most applicable to unfavorable mobility ratios and heterogeneous permeability fields. Although streamlines evolve during a non-unit mobility ratio displacement, for a heterogeneity dominant reservoir, the streamlines evolve little. An important fact is that the order of breakthrough of streamlines is preserved even though length and volume may change. That is, if a streamline passes through a high permeability channel at the start of the displacement process, that channel will remain a channel throughout the entire process. The streamlines with the smallest pore volume or time of flight always break through earliest, no matter how they evolve.

\subsection{Simplifying the Inverse System}

For unit mobility ratio, the inverse system can be simplified by defining relative or normalized parameters such as

$$
\left[\begin{array}{ccccc}
b_{11} & b_{12} & b_{13} & \cdots & b_{1 N} \\
b_{21} & b_{22} & b_{23} & \cdots & b_{2 N} \\
b_{31} & b_{32} & b_{33} & \cdots & b_{3 N} \\
\cdots & & & & \\
b_{N 1} & b_{N 2} & b_{N 3} & \cdots & b_{N N}
\end{array}\right]\left[\begin{array}{c}
\delta k_{1} \\
\delta k_{2} \\
\delta k_{3} \\
\cdots \\
\delta k_{N}
\end{array}\right]=-\left[\begin{array}{c}
e_{t, B T, 1} \\
e_{t, B T, 2} \\
e_{t, B T, 3} \\
\cdots \\
e_{t, B T, N}
\end{array}\right]
$$

where $e_{t, B T, i}$ is normalized error in breakthrough time of streamline $i$ to be defined in Eq. (14), $\delta k_{j}$ is the normalized modification of effective permeability along streamline $j$, and $b_{i j}$ is the derivative of $e_{t_{B T}, i}$ with respect to $\delta k_{j}$. For the purpose of discussing updates from one iteration

to the next, let $k_{j}^{\lambda+1}$ be the new value of $\boldsymbol{k}_{j}$, and likewise, $k_{j}^{\lambda}$ be the previous value. In equation form, the normalized variables are

$$
\begin{aligned}
& e_{t_{B T}, i}=E_{t_{B T}, i} / t_{D, B T, i}^{R}=\left(t_{D, B T, i}^{C}-t_{D, B T, i}^{R}\right) / t_{D, B T, i}^{R} \\
& \delta k_{j}=\frac{k_{j}^{\lambda+1}-k_{j}^{\lambda}}{k_{j}^{\lambda}}=\frac{\Delta k_{j}}{k_{j}^{\lambda}} \\
& b_{i j}=\frac{\partial e_{t_{D, B T, i}}}{\partial\left(\delta k_{j}\right)}=\frac{k_{j}^{\lambda}}{t_{D, B T, i}^{R}} \frac{\partial t_{D, B T, i}}{\partial k_{j}^{\lambda+1}}
\end{aligned}
$$


Substitute Eq. (12) into (16),

$$
b_{i j}=\left\{\begin{array}{l}
\frac{k_{i}}{\sum_{k=1}^{N_{s l}} c_{i k} V_{D, k} k_{k} / k_{i}}\left(-\frac{1}{k_{i}^{2}} \sum_{k=1, k \neq i}^{N_{s l}} c_{i k} V_{D, k} k_{k}\right) \approx-1, \quad \text { if } i=j \\
\frac{k_{i}}{\sum_{k=1}^{N_{s l}} c_{i k} V_{D, k} k_{k} / k_{i}}\left(\frac{c_{i j} V_{D, j}}{k_{i}}\right)=\frac{c_{i j} V_{D, j} k_{i}}{\sum_{k=1}^{N_{s l}} c_{i k} V_{D, k} k_{k}} \approx \frac{1}{N_{s l}}, \quad \text { if } i \neq j
\end{array}\right.
$$

With the normalization above, the elements of the matrix are now functions of only matrix size $N_{s l}$ for a given set of streamlines. Therefore, the inverse of the matrix is also solely a function of $N_{s l}$. As is shown in Eq. (17), when the number of streamlines is large, the offdiagonal terms of the matrix are close to 0 . Therefore, a unit matrix is a good approximation of this non-dimensionalized matrix $[\boldsymbol{B}]$, and therefore inversion of this matrix is also unit. This demonstrates that decoupling of the streamlines is possible. Decoupling of streamlines means that the contribution of a streamline to the error of the production data is mainly related to the average permeability of the streamline. Thus, all of the elements in the inverse of the matrix are calculated directly. This observation is verified by practice as discussed later.

The above simplification works well for unit mobility ratio, as will be demonstrated. For non-unit mobility ratios, it also works to some extent, especially for cases where the mobility ratio is close to unity or heterogeneity is the dominant factor.

In non-unit mobility ratio cases, the elements $\boldsymbol{a}_{i j}$ of matrix $[A]$ are functions of mobility ratio $M$. If we repeat the above process, we do not get a matrix as simple as $[\boldsymbol{B}] \mathrm{in}$ Eq. (17). An alternative for non-unit mobility ratio cases is to solve the system of equations in Eq. (4) by inverting the matrix $[A]$. However, the streamlines evolve during the displacement process. To obtain a good history match with such a procedure, we may need to select several different streamline distributions over the time period of interest. Each of the distributions could be used to match a segment of the fractional flow curve. This is computationally intensive and so we have not implemented this alternative yet. The case studies indicate that it is appropriate to apply the simplification made for unit mobility ratio to non-unit mobility ratio cases and treat streamlines as non-evolving during inverse calculation. 


\section{MATCH OF FLOW RATE AND PRESSURE}

As each streamline carries the same flow rate, we distribute evenly the error of flow rate of a producer to each streamline (or streamtube). The error in pressure drop of the injectorproducer pair is exactly the same as that of each streamline connecting the pair.

We group flow rate and pressure as a single term

$$
F=q / \Delta p
$$

The contribution of each of the streamlines to the error of $\boldsymbol{F}$ for a producer is computed. The error of streamline i, denoted as $\boldsymbol{F}_{\boldsymbol{i}}$, is related to the average permeability of this streamline by Darcy's law. Therefore, the sensitivity of the $\boldsymbol{F}_{\boldsymbol{i}}$ to the average permeability can be computed analytically. Once the error of flow rate and pressure drop_are known, we can readily compute the modification of average permeability of each streamline to minimize the error.

\section{PERMEABILITY UPDATES}

For the forward simulation, we solve the pressure field and streamline distribution as many times as required to obtain convergence, but in the inverse process, we choose one of streamline distributions to compute the streamline properties and update permeability along this streamline distribution. In the inverse process, parameters are non-dimensionalized, therefore, the modification of permeability is performed in a relative manner. That is, we compute a factor that is used to multiply the original permeability value.

The permeability modification for a streamline is the weighted geometric average of the two modifications: one for matching the fractional flow curve and the other for matching flow rate and pressure drop. Our case study shows that equal weighting is acceptable.

Mapping the permeability modifications onto grid blocks is performed in the following way. When the average permeability of a streamline must be multiplied by a factor $f$ to match its breakthrough time, then the permeability of all the grid-blocks along this streamline is multiplied by $f$. This gives us the proper average permeability of the streamline. When more than one streamline passes through the same grid-block, then for this block, the factor for multiplication is the geometric average of all of the factors for the streamlines passing through this block. This leads to inexact modification of the permeability field and the need for iteration. For those grid- 
blocks where no streamlines pass through them, we do not modify their permeability. However, when the number of streamlines is large, these grid-blocks are few.

\section{STEPS OF THE APPROACH}

This approach can be implemented in a relatively simple procedure that involves no modification to the forward streamline simulator, provided that streamline positions are output and flow is evenly distributed among streamlines. This is especially helpful when source code is not available. The steps include:

1. Obtain an initial permeability field by guess or geostatistical realization;

2. Run a streamline simulation on the initial permeability field with the appropriate number of re-computations of the pressure field to obtain a converged forward solution. Check whether the simulation results match the field data (reference data) including fractional-flow curve at the producers, flow rate, and pressure. If not, modify the permeability according to the following steps;

3. Work on the streamlines. Calculate the time of flight (or the associated pore volume) for all streamlines. Sort the streamlines in ascending order of pore volume;

4. Compute the difference in fractional flow, flow rate, and pressure between the simulation result and the reference. Relate differences in the fractional-flow curve to the corresponding streamline;

5. Solve the system described above (either Eq. (13) or Eq. (4)) to compute the modification of the effective permeability of each streamline required for a good match to available data;

6. Map the modification at the streamline level onto grid-blocks honoring the effective permeability of each streamline;

7. Iterate steps 2 to 6 until a satisfactory match is achieved.

\section{EXAMPLE APPLICATIONS}

Several cases have been tested with this approach and the results show that this method is robust with the stated assumptions and converges quickly. Four cases are presented below. First, a reference permeability field is generated, and then 3DSL run with constant injection rate, except for the last case, to obtain the reference production data. Water cut and pressure drop are the data from the reference case that we try to match. An initial permeability field is guessed to 
start the procedure. The permeability field is either uniform or generated by a geostatistical sequential Gaussian simulation method (Deutsch and Journel, 1998) where the field is conditioned to sparsely distributed data. For simplicity, all the cases deal with a quarter of a five-spot pattern. Our formulation is not restricted to this pattern, nor is the method restricted to two-dimensional areal cases. For all of the cases, the injector is located at the lower left corner, and the producer at the upper right corner of the pattern. The pressure at the producer is constant and the fluid is incompressible.

\subsection{Large Scale Trends, Cases 1 and 2}

The two cases discussed in this subsection show how a large-scale trend is retrieved. By large scale, we mean a permeability feature that spans nearly from injector to producer. For both cases, the mobility ratio is unity, the water injection rate is constant, and the initial guess for the permeability field is uniform. The numerical solution of the Buckley-Leverett equation is mapped along the streamlines.

The relative permeability functions used for these two cases are described by the following expressions:

$$
\begin{aligned}
& k_{r w}=S_{w} \\
& k_{r o}=S_{o}
\end{aligned}
$$

Viscosity is $1 \mathrm{cp}$ for both phases. Thus, we obtain piston-like displacement along streamlines.

In Case 1, the reference permeability field contains a high permeability channel connecting the injector and producer as shown in Fig. 1(a). In Fig. 1, light gray indicates low permeability and dark gray high permeability. Starting from a uniform permeability field of 500 md, four iterations are required to match water-cut and pressure data. Each iteration includes running the flow simulation, computing errors, modifying the permeability of the streamlines, and mapping the modification onto the grid-blocks. The inferred permeability field is shown in Fig. 1(b). Although somewhat more diffuse, the feature linking injector and producer is reproduced. Figure 2 shows the evaluation of the match of water-cut, and Fig. 3 shows the normalized error for fractional flow and pressure. After four iterations, the match is very good. The inversion converges quickly and the relative error is less than $1 \%$. Most of this error is associated with the match of the water-cut curve. The computational time is spent mainly in running the forward flow simulation. The inversion requires only a few seconds because the solution of the inverse system of equations is simplified. 
The configuration of Case 2 is similar to that in Case 1, except in the orientation of features in the permeability distribution. In the first case, permeability features were aligned along flow paths between injector and producer. In Case 2, an off-flow-trend barrier exists as shown in the reference permeability field of Fig. 4a. With the same procedure as in Case 1, a very good match to fractional flow and pressure behavior is achieved with two iterations as illustrated in Figs. 5 and 6. However, the geometry and size of the barrier are not reproduced very well. Comparing the reference permeability and the inferred permeability distributions in Fig. $4 \mathrm{a}$ and $4 \mathrm{~b}$, the inferred field has a diffuse low-permeability region that nearly spans from the injector to the producer. This is the worst case we can formulate to test reproduction of a true permeability field. The reason for this result and some possible solutions are explored in the discussion.

\subsection{Effect of Mobility Ratio, Case 3}

In this case, we infer the permeability field by matching the results of a tracer injection test. The well configuration is the same as Case 1 as are the relative permeability curves and viscosities. A large slug of tracer of size $0.6 \mathrm{PV}$ is injected. In forward flow simulations, the Buckley-Leverett solution is again mapped along the streamlines.

To generate more realistic reference and initial permeability fields, two permeability field realizations shown in Fig.7 were generated by the routine SGSIM (Deutsch and Journel, 1998) based on scattered permeability data with similar direction of continuity. One realization, Fig. $7 \mathrm{a}$, is used as the reference, the other, Fig. 7b, is used as the initial permeability field. The two realizations are quite different and the flow simulation results from these two permeability fields show large obvious differences in production response as illustrated in Fig. 8b. Note that the realizations are not constrained by production data.

Our method requires monotonic increase of tracer concentration or water cut. For this slug injection, we perform superposition on the reference production data_before going to the inverse process. In general, a tracer response concentration curve is the result of subtraction of a solution of continuous injection that begins when tracer injection ends from a solution of continuous injection beginning at the start of tracer injection. We retrieve the monotonically increasing production curve from observed tracer data by adding back the part subtracted as shown in Fig. 8a. This can be done easily because the concentration profiles of the two 
continuous injection solutions are identical in dimensionless time. More accurately, they are identical solutions shifted by a value of slug pore volume on the dimensionless time axis. Therefore, the monotonically increasing tracer concentration solution can be deduced solely from the observed tracer concentration by the de-superposition approach. Brigham and AbbaszadehDehghani $^{[30]}$ discuss these concepts in greater detail.

Six iterations were performed to match the reference tracer concentration data, and the results are illustrated in Figs. 7 to 9. The match is excellent in terms of reproducing tracer concentration, Fig.8b, and pressure characteristics. Figure 9 shows that most of the error remains in matching the tracer concentration curve, but this error is less than $2 \%$. The match of permeability distribution is quite satisfactory, as shown by comparing Fig. 7 (a) and (c). Note that the inferred permeability field is the best that we can achieve given the producer information and initial guess without further information to constrain the inversion. The treatment of slug injection by superposition of the Buckley-Leverett solution along streamlines is embedded in the inverse process. This case shows that this approach serves our purpose well.

The match obtained in Fig. 7c, was tested with two-phase displacement for other non constant mobility ratios. Flow simulations were run on the reference and inferred permeability fields at end-point mobility ratios of 0.25 and 5 , respectively.

The relative permeabilities used are described by the following expressions

$$
\begin{aligned}
& k_{r w}=k_{r w}^{0}\left(\frac{S_{w}-S_{w c}}{1-S_{o r}-S_{w c}}\right)^{2} \\
& k_{r o}=k_{r o}^{0}\left(\frac{1-S_{o r}-S_{w}}{1-S_{o r}-S_{w c}}\right)^{2}
\end{aligned}
$$

where the end point relative permeabilities for oil and water are set to $k_{r o}^{0}=0.8$ and $k_{r w}^{0}=0.2$. The connate water saturation and residual oil saturation used are $S_{w c}=0.2, S_{o r}=0.2$. Water viscosity is $1 \mathrm{cp}$. The viscosity of oil is $1 \mathrm{cp}$ for favorable mobility ratio $(\mathrm{M}=0.25)$ and $20 \mathrm{cp}$ for unfavorable mobility ratio $(\mathrm{M}=5)$.

Figure 10 shows the comparison. Also given is the water-cut curve obtained for the initial permeability field. It indicates that the permeability field inferred by matching production data at one mobility ratio can be used to predict displacement processes at other mobility ratios. 
This is very useful when a tracer study is used to characterize reservoir heterogeneity and the inferred permeability distribution is then used to predict the reservoir's future performance.

\subsection{Variable Injection Rate, Case 4}

In this case, the injection rate undergoes several step changes during the course of the displacement process. The fluids are again incompressible. The endpoint mobility ratio is 2.5 , requiring multiple pressure solves (Vasco et al., 1998) in the forward solution. The relative permeability functions are described by Equation 20 as in Case 3, and viscosities for water and oil are $1 c p$ and $10 c p$, respectively. Two permeability fields are generated by sequential Gaussian simulation. (Lake, 1989). One is used as a reference and the other as an initial permeability field as shown in Fig. 11a and b. In the inversion, the streamline distribution from the first pressure solve is chosen to compute the properties of streamlines and the permeability modifications.

With the procedure given above and fixing the injection rate schedule to match the history of the reference case, a very good match to the fractional flow curve and the pressure at the injector is obtained as shown in Figs. 12 and 13, respectively. The error during iteration is given in Fig. 14. Only three iterations are needed to converge despite the relatively complicated injection conditions. The inferred permeability field is shown in Fig. 11c. Visually the final match appears to be closer to the reference permeability field of Fig. 11a than is the initial permeability in Fig. 11b.

\section{DISCUSSION}

The example applications show that this approach is robust within the stated limitations and converges quickly. Each iteration significantly reduces the errors in fractional flow, pressure drop, and flow rate. In all of the examples, an acceptable match to the production data is obtained within a small number of iterations. One strength of this approach is that the input of the inverse problem is simply the usual output of the streamline simulation. Namely, streamline coordinates, production data including fractional flow, flow rate, and pressure. Therefore, this approach stands alone. Importantly, it appears to be expandable to integrate geostatistical data or to honor geological and seismic data. We are actively pursuing this task and discuss this topic below. 
Another strength is that repeated inversion of a matrix is not needed for the cases studied. The permeability modification for every iteration is computed directly based on differences between the streamline breakthrough time from field observation and the simulation result. Therefore, this process takes very little computational time. All of the examples illustrated here used the simplified inverse approach derived for unit mobility ratio cases. It appears that this idea works well for flow with unfavorable mobility ratios in heterogeneous reservoirs. Further study is required.

The computation of the error in water-cut for both unit and non-unit mobility ratio cases is performed using fractional-flow curves from the simulation and the reference results. The pore volume associated with each streamline is calculated for the purpose of sorting streamlines. The accuracy of computing streamline pore volume does not affect inversion results as long as the correct ordering of the breakthrough time is obtained. Therefore, for non-constant, non-unit mobility ratio cases, the method works well as long as the order of streamline breakthrough time is preserved, even though the streamlines evolve.

Streamline permeability modifications are mapped directly to grid-blocks by propagating the indicated change through all of the grid-blocks that a particular streamline passes. For example, when the effective permeability of a streamline needs to be increased by 5 percent, then the permeability of all the grid-blocks along that streamline are increased by 5\%. Mapping is not unique and different mapping schemes can produce the same effective streamline permeability. Necessarily, however, the water-cut curve is matched and the inferred field can be used to predict results of other mobility ratios. In the mapping process, it is possible to enforce constraints to honor observed geological information, if any.

Inverse solutions can be constrained in many different ways. Three approaches, as described below, appear to be consistent with this approach.

A simple, but probably inefficient, method is to generate a number of permeability fields by sequential Gaussian simulation based on available geological information, and to check whether the effective permeability distribution from a particular realization matches that from the inversion. The check can be performed in the following way. Solve the pressure field and the streamline distribution once for permeability field from geostatistical simulation. A unit mobility ratio should suffice. Sort the streamlines in the order of breakthrough time as we did for the inverse process. Compute the effective permeability and time of flight of each streamline, 
compare it with the effective permeability and time of flight from the inversion. In this manner, realizations are sorted. Those realizations within an acceptable value of the error in permeability are expected to generate a good match in production response, and therefore are accepted. It is easy to generate geostatistical realizations and solve the pressure field once to obtain streamline distribution. This approach requires much less computational work than running a full flow simulation on each realization.

Another way to constrain the inversion is to obtain a map of the expected pattern of highs and lows in the permeability field based on geological or seismic information. The high and low values would only be relative. When mapping the permeability modification from streamlines to grid-blocks, the decision of whether to modify the permeability of a grid-block could be based on whether the permeability is to be increased or decreased, and whether this change agrees with the geological information. Thus, permeability could be mapped to honor prior information about the field.

A third approach is a combination of this work with the master-point idea (Wen et al., 1998). We could compute the modification of the average permeability, and convert this modification to a number of pre-selected master points (i.e, grid-blocks). Kriging is performed to propagate the modification at the master points to other grid-blocks. When kriging, we incorporate known geological information, such as direction of continuity and correlation length, and all the grid-blocks are reached for modification.

Examples shown here are for two-dimensional flow problems. We continue to work on the generalization to three-dimensional problems with gravity. Additionally, this approach was originally designed for incompressible two-phase flow or tracer flow studies. That is, the fractional flow curve or tracer breakthrough curve at the producers is required to infer the heterogeneity of the reservoir. More study is needed to extend this procedure to compressible single-phase flow scenarios such as well test problems. Also, streamline simulation encounters difficulties when infill wells are drilled, injectors are converted to producers, or vice versa, well completions are changed, or radical changes are made in pattern balancing. In forward flow simulation, such actions require that_streamlines be recomputed. In history matching, multiple realizations of streamline geometry would have to be honored. This is an important topic, but we must address it later. 
In Case 1, the permeability feature is on trend, and we find that it is easy to retrieve the features of the distribution. However, if the heterogeneity is off trend, then the permeability feature may not be retrieved as easily by this approach. Case 2, as shown in Fig. 4, indicates that the off-trend barrier is reproduced as diffuse, scattered regions of relatively low permeability if no constraints of mapping are enforced. This result occurs because all of the grid-blocks along a streamline are multiplied by the same factor during the update of permeability. This problem can be overcome by applying geological information while mapping, as discussed, or generating a better initial permeability field. Nevertheless, the effective permeability of each streamline in the inferred permeability field is very close to that of the corresponding streamline in the reference permeability field. As a result, the fractional flow curve and pressure drop obtained for the inferred permeability field are quite close to the reference values.

Results, not shown here, indicate that the inverse solutions are very sensitive to relative permeability. It is necessary to obtain accurate relative permeability curves in order to retrieve a good inverse solution. This is true of most history-matching approaches. As long as we use the same relative permeability curves in both the forward simulation and inverse process, the inferred permeability field will give us a good history match.

Actual production data is never as smooth as that in our synthetic examples. In general, for continuous injection of one phase to displace another phase, the fractional flow of the injected phase at the producers is monotonically increasing. However, noise may cause it, apparently, not to be so. In such cases, we need to preprocess the data. The first step would be to use dimensionless time (pore volume) rather than real time. To reduce the effect of noise, a filter to remove small time scale variations (high frequency) is needed, while maintaining the variations in large time scale. Normally, noise is random and therefore does not have a large time scale trend. In general, it is the bulk reservoir properties that are important for predicting reservoir performance, and they are represented in large time scale variations. Therefore, removing small time scale variation, even if it is not caused by noise, has small effect for prediction. 


\section{CONCLUSIONS}

This approach relates producer water-cut curves to the breakthrough time of individual streamlines. The effective permeability along streamlines is modified directly to history-match the fractional flow curve, pressure drop, and flow rate information. No matrix inversion is involved in the inverse process and therefore it is quite fast. The forward flow simulation with 3DSL is also fast and so, for the examples examined, the entire process appears to be computationally efficient.

The current work examined 2-D areal porous media, where the effect of gravity is not important, as well as incompressible two-phase flow. As with most history-matching approaches, high-quality noise-free data is needed for fast and accurate inversion. This approach works well for reservoirs where heterogeneity is a dominant factor. It also works well for unfavorable mobility ratios because the effects of heterogeneity are exacerbated by the unstable displacement. Although streamlines evolve for non-unit mobility ratio cases during the displacement process, we find it feasible to choose one streamline distribution and apply the simplified inverse system during the inversion.

Importantly, a permeability field inferred by matching production data for one mobility ratio can be used to predict reservoir performance for displacement processes at other mobility ratios.

\section{NOMENCLATURE}

A cross-sectional area of a streamtube, $\mathrm{L}^{2}$

c constant

E Absolute error

$e \quad$ Relative error

$f_{w} \quad$ fractional flow of water

$k$ permeability, $\mathrm{L}^{2}$

$k_{r w} \quad$ relative permeability of water

$k_{r o} \quad$ relative permeability of oil

$l \quad$ streamline length

$M \quad$ mobility ratio

$p \quad$ pressure, $\mathrm{M} /\left(\mathrm{Lt}^{2}\right)$ 


$$
\begin{array}{ll}
q & \text { flow rate, } \mathrm{L}^{3} / \mathrm{t} \\
t_{D} & \text { dimensionless time } \\
V & \text { pore volume, } \mathrm{L}^{3} \\
V_{P} & \text { pore volume } \\
V_{D} & \text { ratio of pore volume } \\
x_{D} & \text { dimensionless length } \\
\phi & \text { porosity }
\end{array}
$$

\section{Subscripts:}

n producer index

$\mathrm{i}, \mathrm{j}, \mathrm{k}$ streamline index

sl streamline

BT breakthrough

D dimensionless

\section{Superscripts:}

C Computed

R Reference

$\lambda$ iteration index

\section{REFERENCES}

1. Batycky, R. P., Blunt, M. J., and Thiele, M. R.: "A 3D Field-Scale Streamline-Based Reservoir Simulator,” SPERE (November 1997) 246-254.

2. Blunt, M.J., Liu, K., and Thiele, M.R.:"A Generalized Streamline Method to Predict Reservoir Flow," Petroleum Geoscience (1996) 2, 259-269.

3. Brigham, W. F. and Abbaszadeh-Dehghani, M., "Tracer Testing for Reservoir Description," JPT (May 1987) 519-527.

4. Chu, L., Reynolds, A. C. and Oliver, D. S.: "Computation of Sensitivity Coefficients for Conditioning the Permeability Field to Well-Test Pressure Data," In Situ , (1995) 19(2), 179-223.

5. Deutsch, C. V. and Journel, A. G., GSLIB, Geostatistical Software Library and User's Guide, Second Edition, Oxford University Press, New York (1998). 
6. Dykstra, H. and Parsons, R.L., "The Prediction of Oil Recovery by Waterflood," Secondary Recovery of Oil in the United States, Principles and Practice, 2d ed., American Petroleum Institute (1950), 160-174.

7. Fay, C. H. and Prats, M.. "The Application of Numerical Methods to Cycling and Flooding Problems" Proceedings of the 3rd World Petroleum Congress (1951).

8. Grinestaff, G.H.: "Waterflood Pattern Allocations: Quantifying the Injector to Producer Relationship with Streamline Simulation," paper SPE 54616 presented at the 1999 Western Regional Meeting, Anchorage, Alaska, 26-28 May.

9. Gupta, A. D., Vasco, D. W. and Long J.C.S.: "Detailed Characterization of Fractured Limestone Formation Using Stochastic Inverse Approaches," SPE Ninth Symposium (1994).

10. Hewett, T.A. and Behrens, R.A.: "Scaling Laws in reservoir Simulation and Their Use in a Hybrid Finite Difference/Streamtube Approach to Simulation the Effects of Permeability Heterogeneity," in Reservoir Characterization, II, L. Lake and H.B.J. Carroll (eds.), Academic Press Inc., London (1991) 402-441.

11. Hewett, T. A., and Yamada, T.: "Theory of the Semi-Analytical Calculation of Oil Recovery and Effective Permeabilities Using Streamlines," Advances in Water resources (1997) 20(5-6), 279-295.

12. Higgins, R.V., Boley, D.W. and Leighton, A.J.: "Aids to Forecasting the Performance of Water Floods," JPT (September 1964) 1076-1082.

13. Higgins, R.V. and Leighton, A.J.: "A Computer Method to Calculate Two-Phase Flow in Any Irregularly Bounded Porous Medium," JPT (June 1962) 679-683.

14. King, M.J., and Datta-Gupta, A.: "Streamline Simulation: A Current Perspective," In Situ, 22(1), (1998) 91-140.

15. Lake. L. W., Enhanced Oil Recovery, Prentice Hall, Englewood Cliffs, NJ (1989).

16. Landa, J. L. and Horne, R. N.: "A Procedure to Integrate Well Test Data, Reservoir Performance History and 4-D Seismic Information," paper SPE 38653 presented at 1997 Annual Technical Conference and Exhibition in San Antonio, Texas, 5-8 October.

17. Lepine, O. J., Bissell, R. C., Aanonsen, S. I., Pallister, I. and W Barker, J.: "Uncertainty Analysis in Predictive Reservoir Simulation Using Gradient Information," SPE 48997, proceedings of the SPE Annual Meeting, New Orleans (September 1998).

18. Martin, J.C. and Wegner, R.E.: "Numerical Solution of Multiphase, Two-Dimensional Incompressible Flow Using Streamtube Relationships," SPEJ (October 1979) 313-323. 
19. Peddibhotla, S., Datta-Gupta, A., and Xue, G.: "Multiphase Streamline Modeling in Three Dimensions: Further Generalizations and a Field Application," paper SPE 38003 presented at 1997 Reservoir Simulation Symposium in Dallas, Texas, 8-11 June.

20. Portella, R. C. M. and Hewett, T. A.: "Fast 3-D Reservoir Simulation and Applications Using Streamlines," paper SPE 39061 presented at the Fifth Latin American and Caribbean Petroleum Engineering Conference and Exhibition in Rio de Janeiro, Brazil, 30 August-3 September 1997.

21. Rahon, D., Edoa, P. F. and Masmoudi, M.: "Identification of geological shapes in reservoir engineering by history matching production data," SPE 48969, proceedings of the SPE Annual Meeting, New Orleans (September 1998).

22. Thiele, M. R., Batycky, R. P., Blunt, M. J. and Orr Jr, F. M.Jr. "Simulating Flow in Heterogeneous Systems Using Streamtubes and Streamlines," SPERE (February 1996) 512.

23. Thiele, M.R., Blunt, M.J., and Orr, F.M. Jr.: "Modeling Flow in Heterogeneous Media Using Streamtubes--I. Miscible and Immiscible Displacements," In Situ (August 1995a) 19(3), 299-339.

24. Thiele, M.R., Blunt, M.J., and Orr, F.M. Jr.: "Modeling Flow in Heterogeneous Media Using Streamtubes--II. Compositional Displacements," In Situ (1995b) 19(4), 367-391.

25. Vasco, D.W. and Datta-Gupta, A.: "Integrating Multiphase Production History in Stochastic Reservoir Characterization," SPEFE (September 1997) 149-156.

26. Vasco, D.W., Yoon, S. and Datta-Gupta, A.: "Integrating Dynamic Data Into HighResolution Reservoir Models Using Streamline-Based Analytic Sensitivity Coefficients," paper SPE 49002 presented at 1998 Annual Technical Conference and Exhibition in New Orleans, Louisiana, 27-30 September.

27. Wang, Y.D., Kovscek, A. R. and Brigham, W.E.: "Effect of Mobility Ratio on Pattern Behavior of a Homogeneous Porous Media", In-Situ, 23(1) (1999) 1-20.

28. Wen, X.H., Clayton, D.V. and Cullick A.S.: "High Resolution Reservoir Models Integrating Multiple-Well Production Data," SPEJ (December 1998) 344-355.

29. Wen, X.H., Clayton, D.V. and Cullick A.S.: "Integrating Pressure and Fractional Flow Data in Reservoir Modeling with Fast Streamline-Based Inverse Method," paper SPE 48971 presented at 1998 Annual Technical Conference and Exhibition in New Orlean, Louisiana, 27-30 September.

30. Wu, Z., Reynolds, A. C. and Oliver, D. S.: "Conditioning Geostatistical Models to TwoPhase Production Data," SPEJ (June 1998) 142-155. 


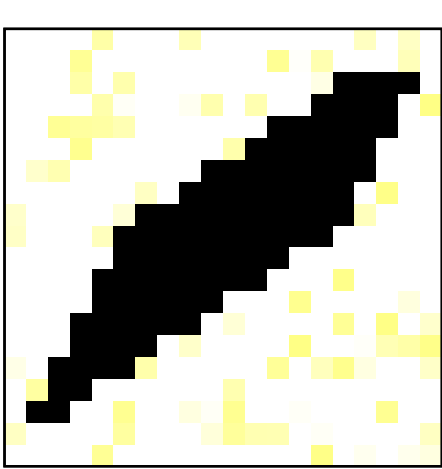

(a)

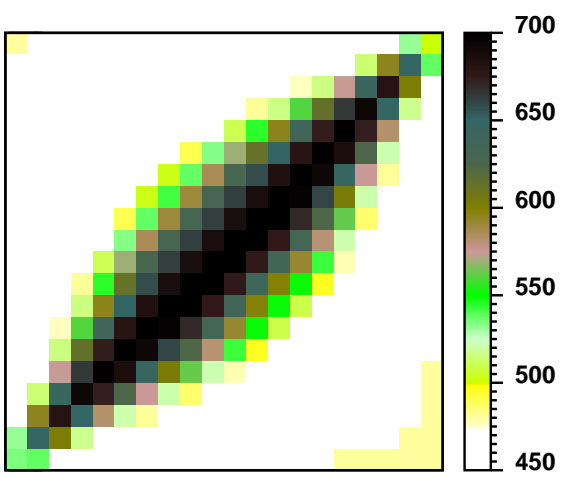

(b)

Fig. 1 Case 1, comparison of permeability fields

(a) reference field and (b) history-matched field 


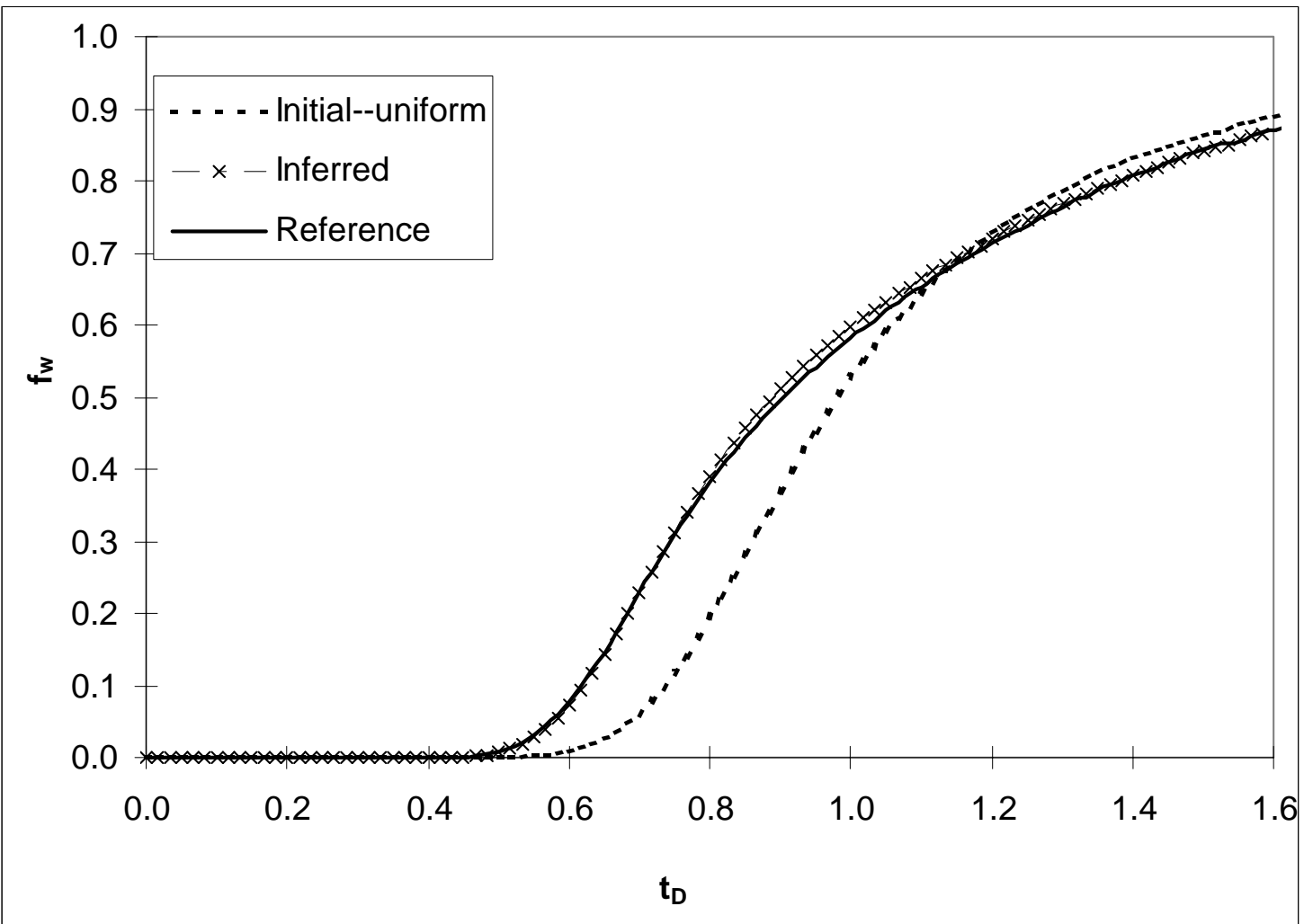

Fig. 2 Case 1, fractional flow curves 


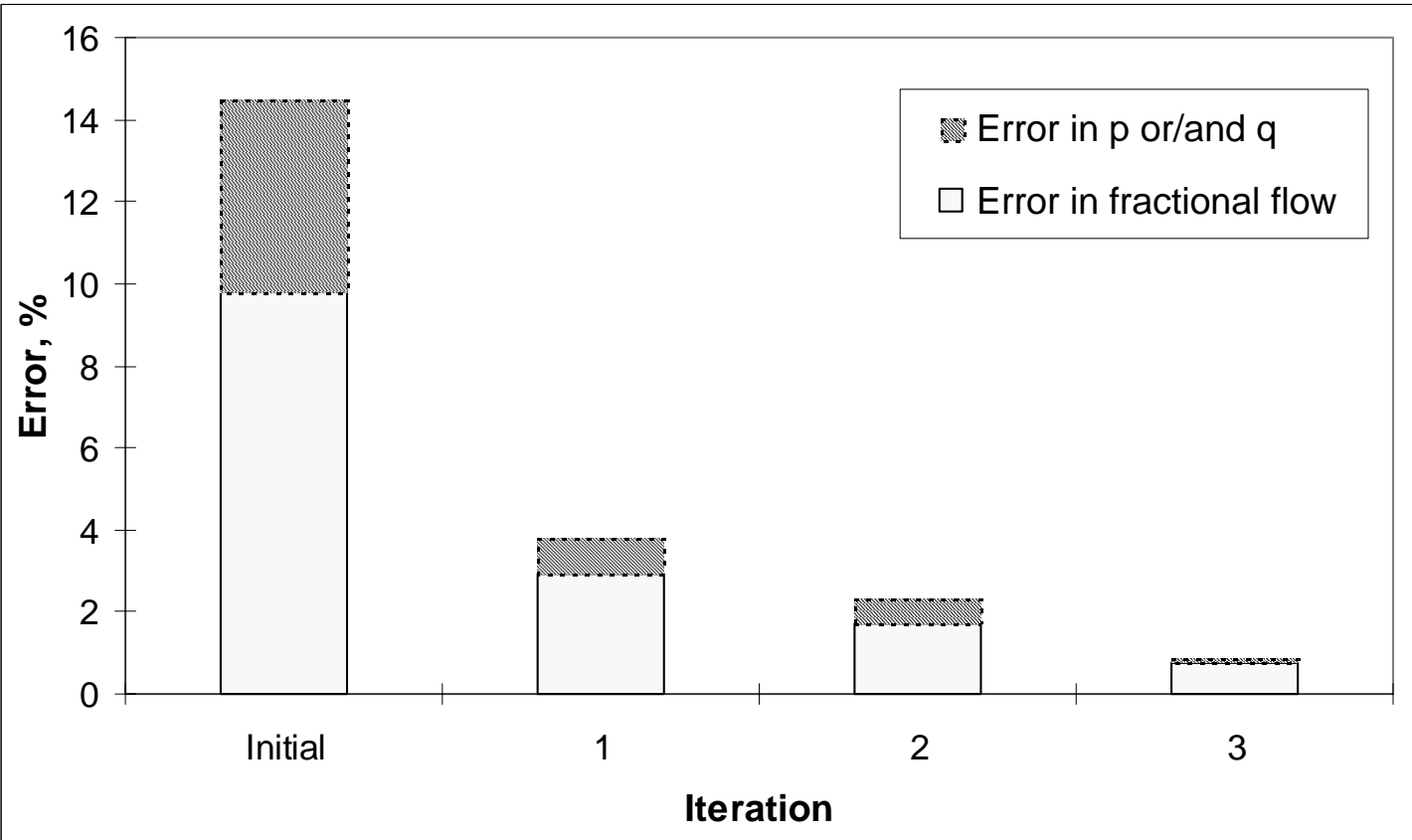

Fig. 3 Case 1, error reduction 


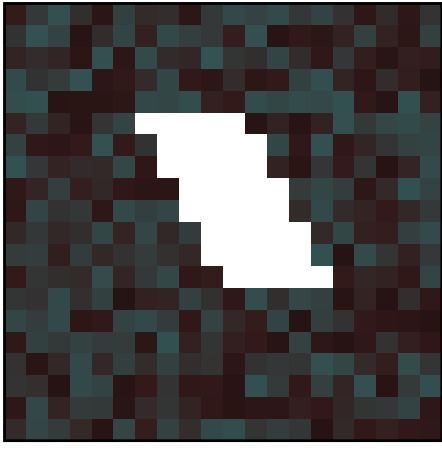

(a)

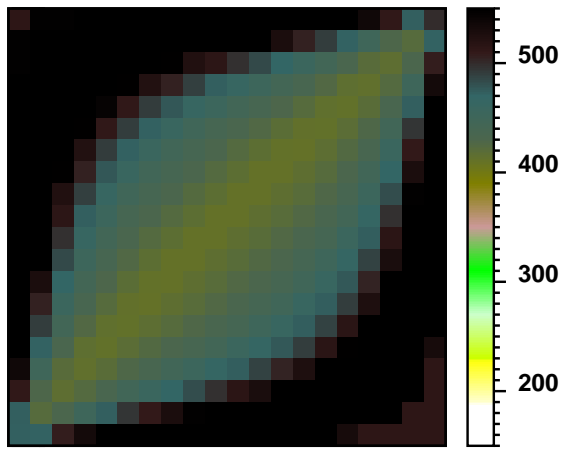

(b)

Fig. 4 Case 2, comparison of permeability fields

(a) reference field and (b) history-matched field 


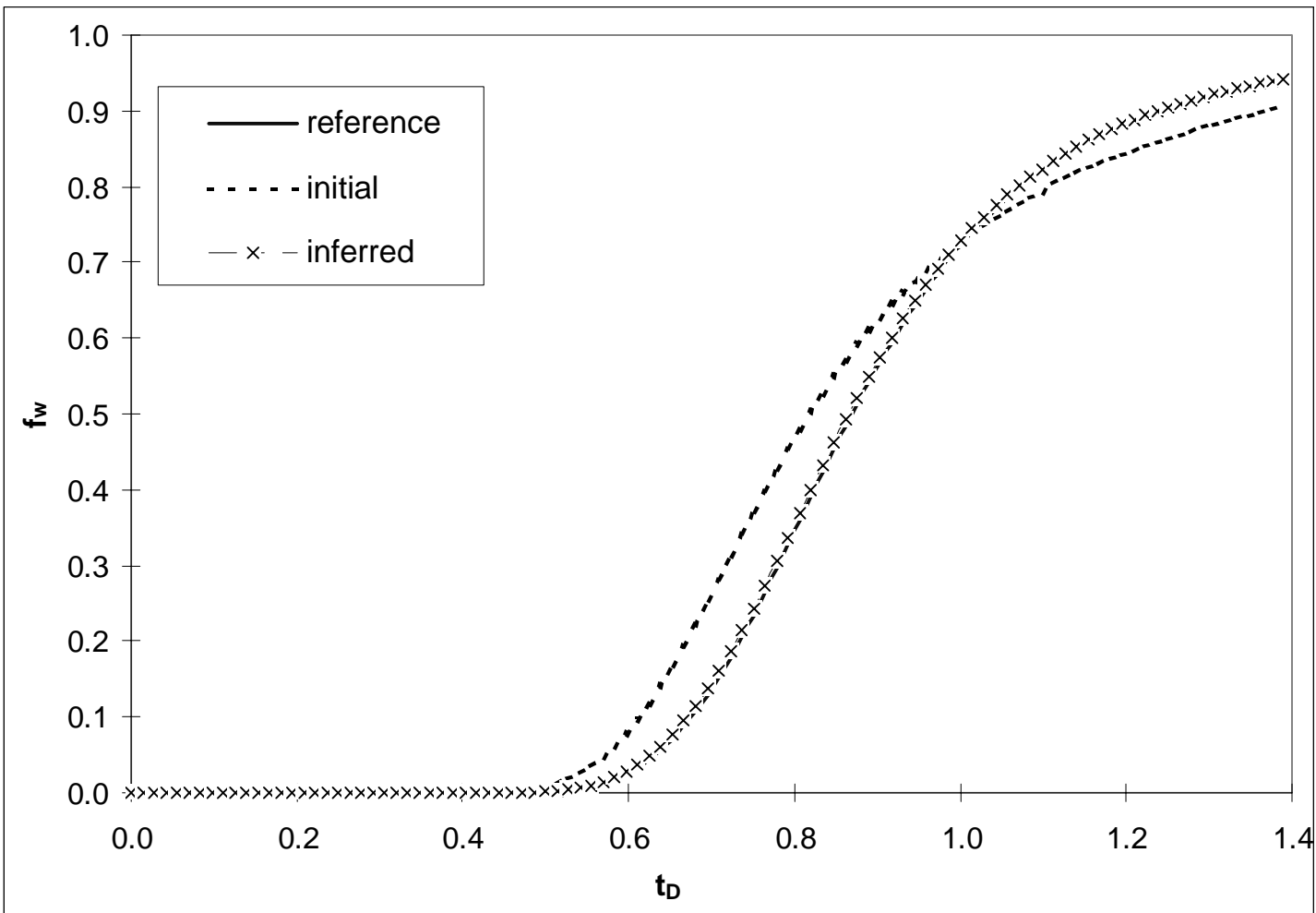

Fig. 5 Case 2, fractional flow curves 


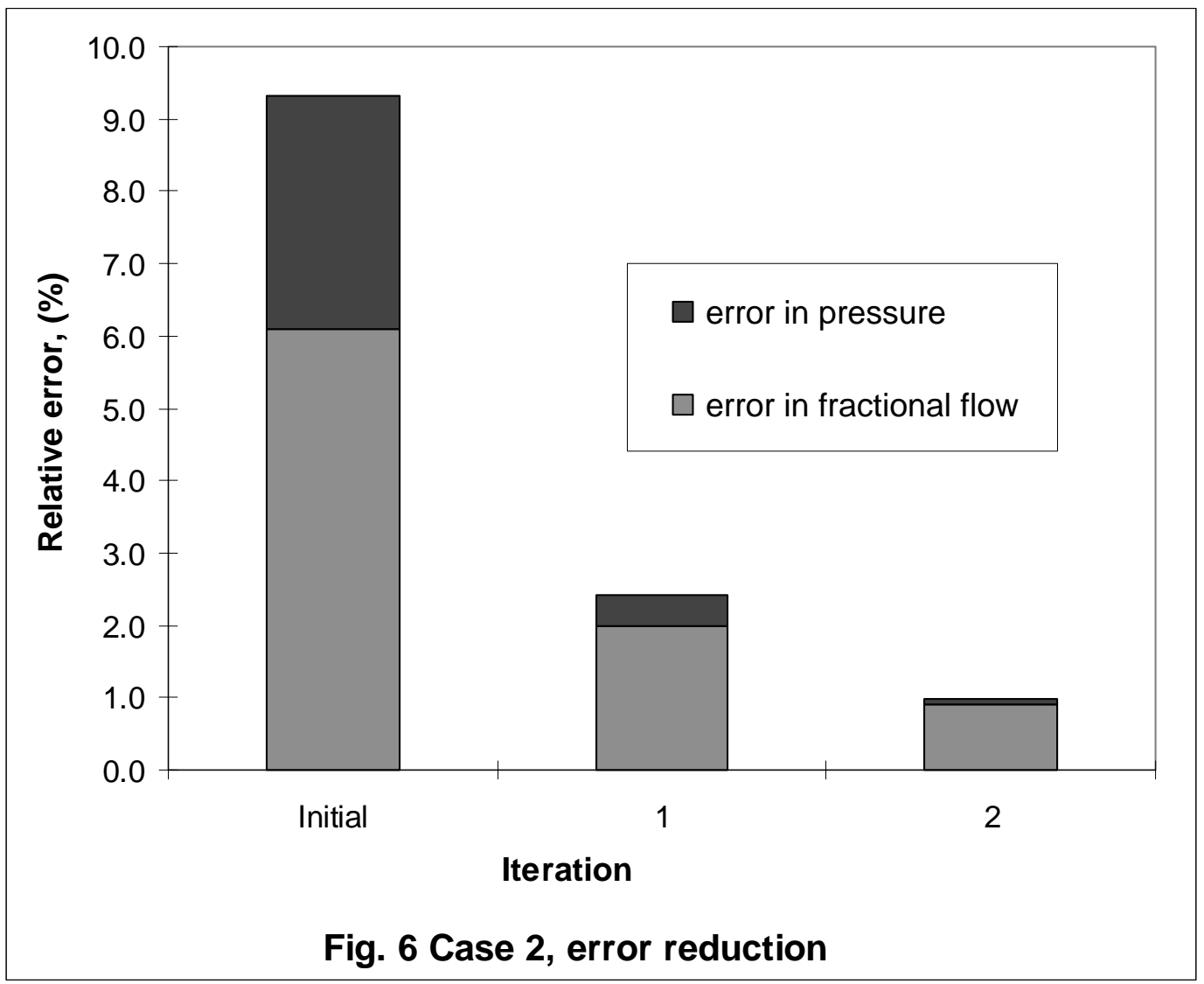




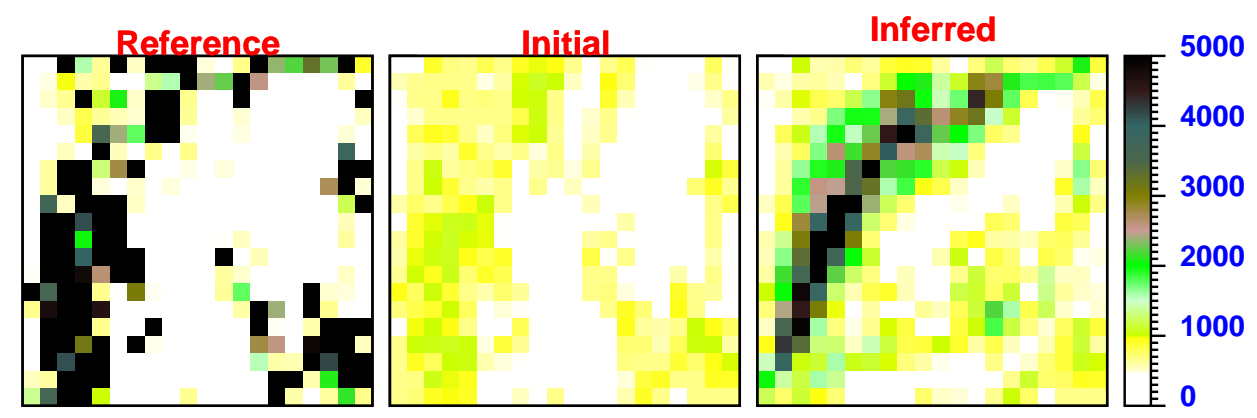

Fig. 7 Case 3, comparison of permeability fields

(a) reference, (b) initial and (c) history-matched field 

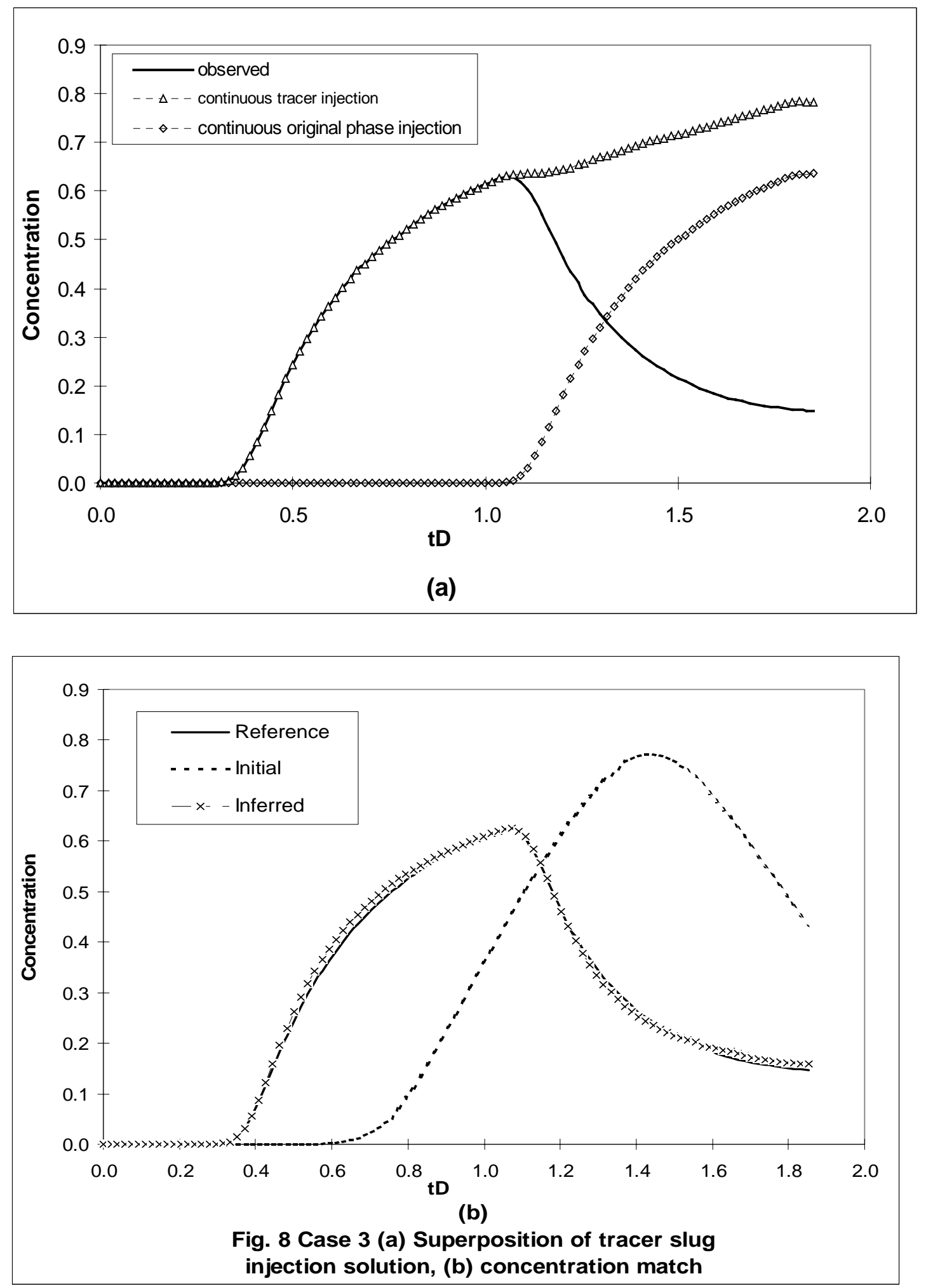


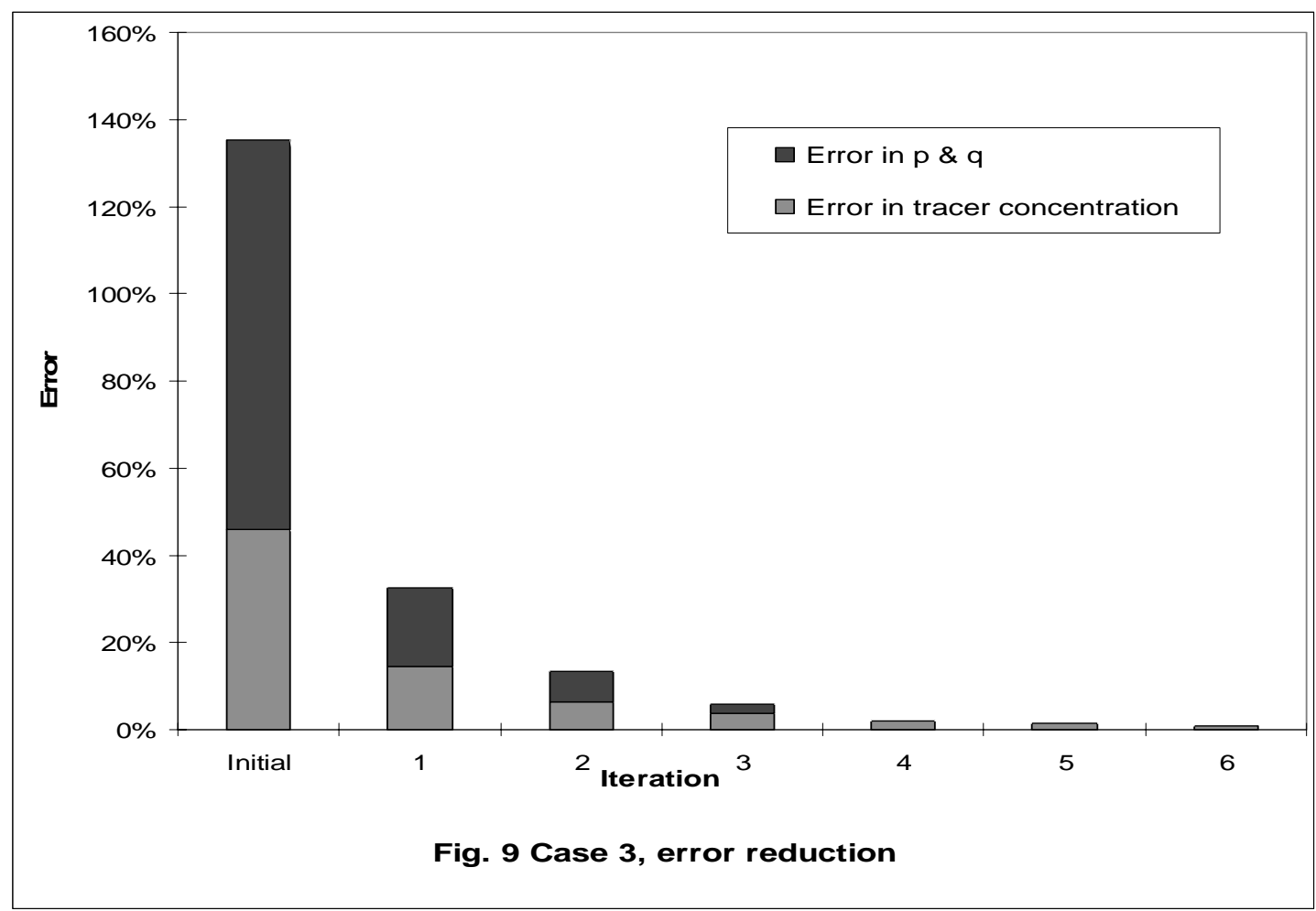




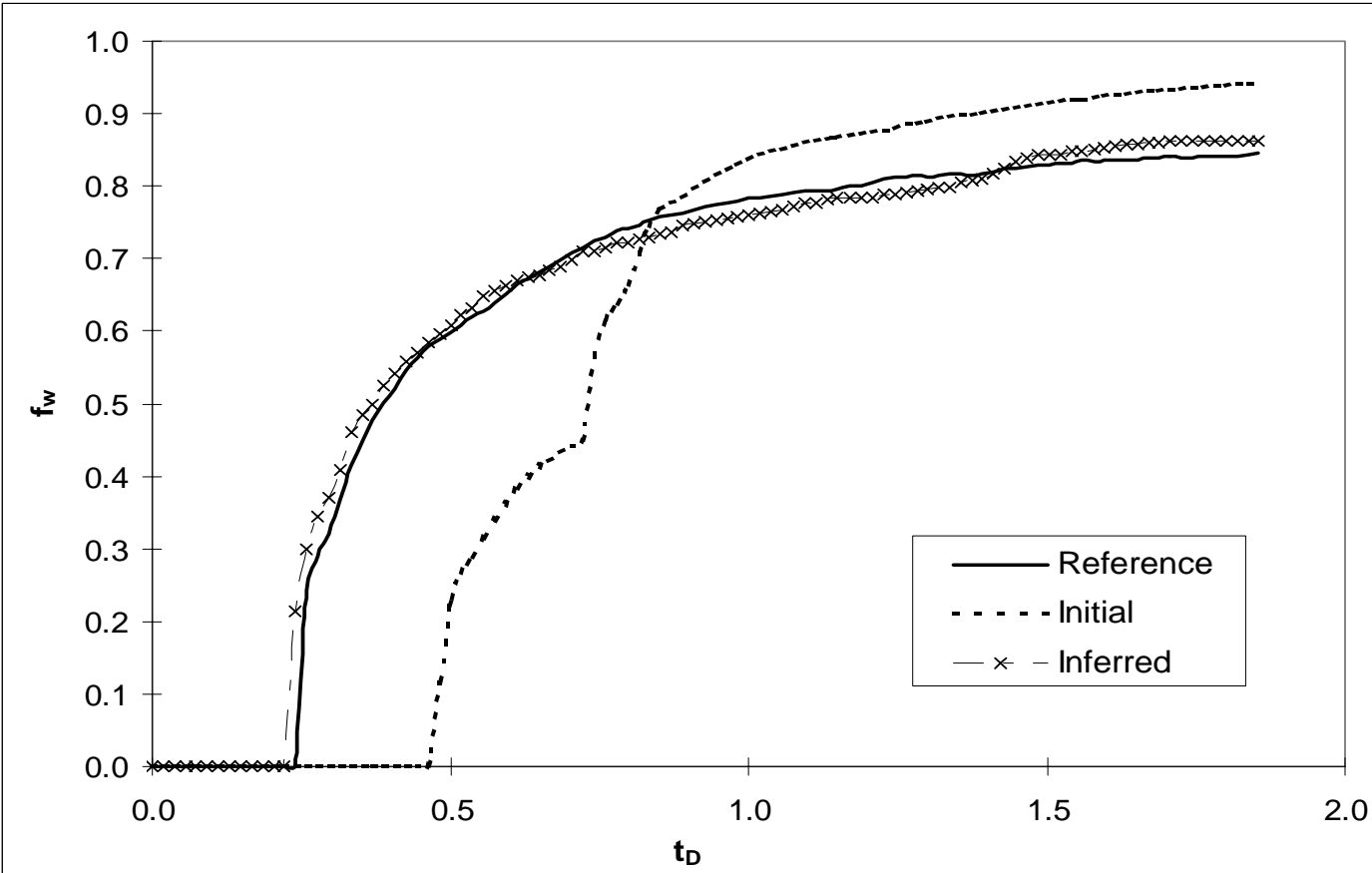

(a)

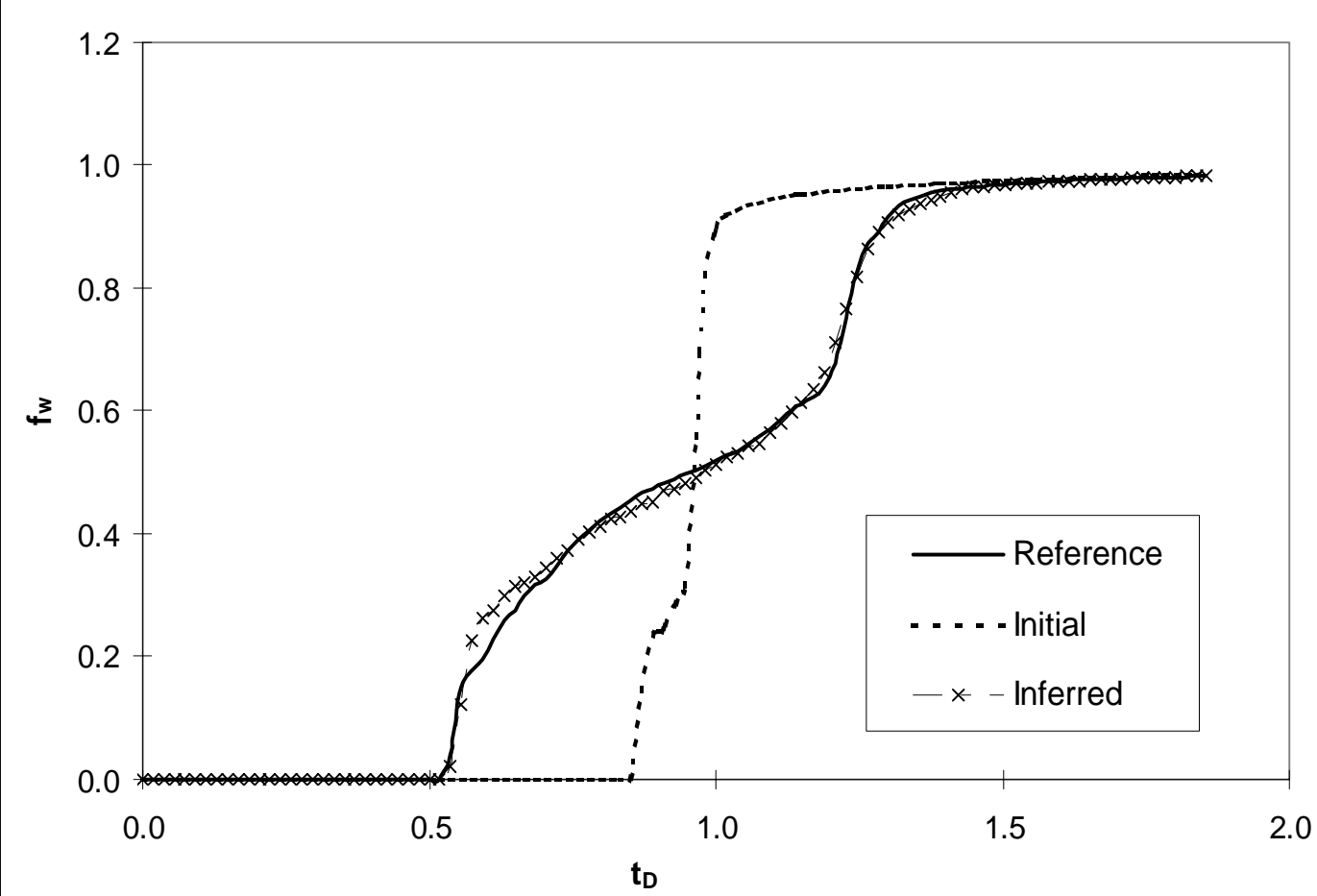

(b)

Fig. 10 Case 3, fractional flow curves(a) $M=5$ and (b) $M=0.25$ 


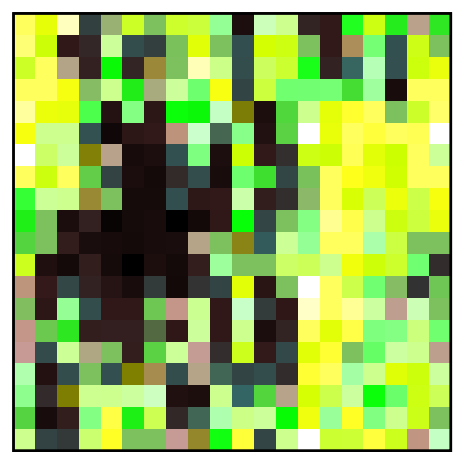

(a)

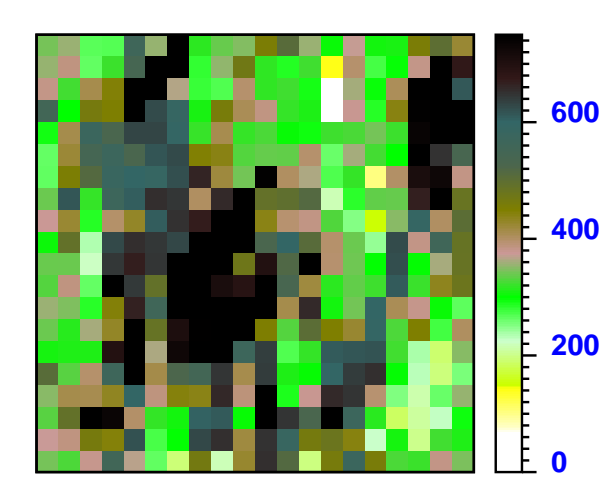

(c)

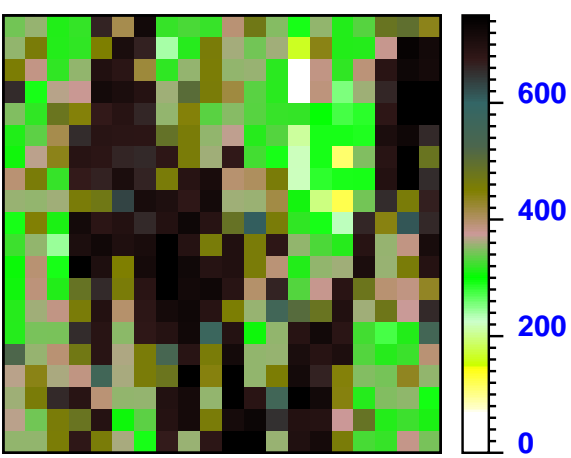

(b)

Fig. 11 Case 4, comparison of permeability fields

(a) reference, (b) initial, and (c) history-matched field 


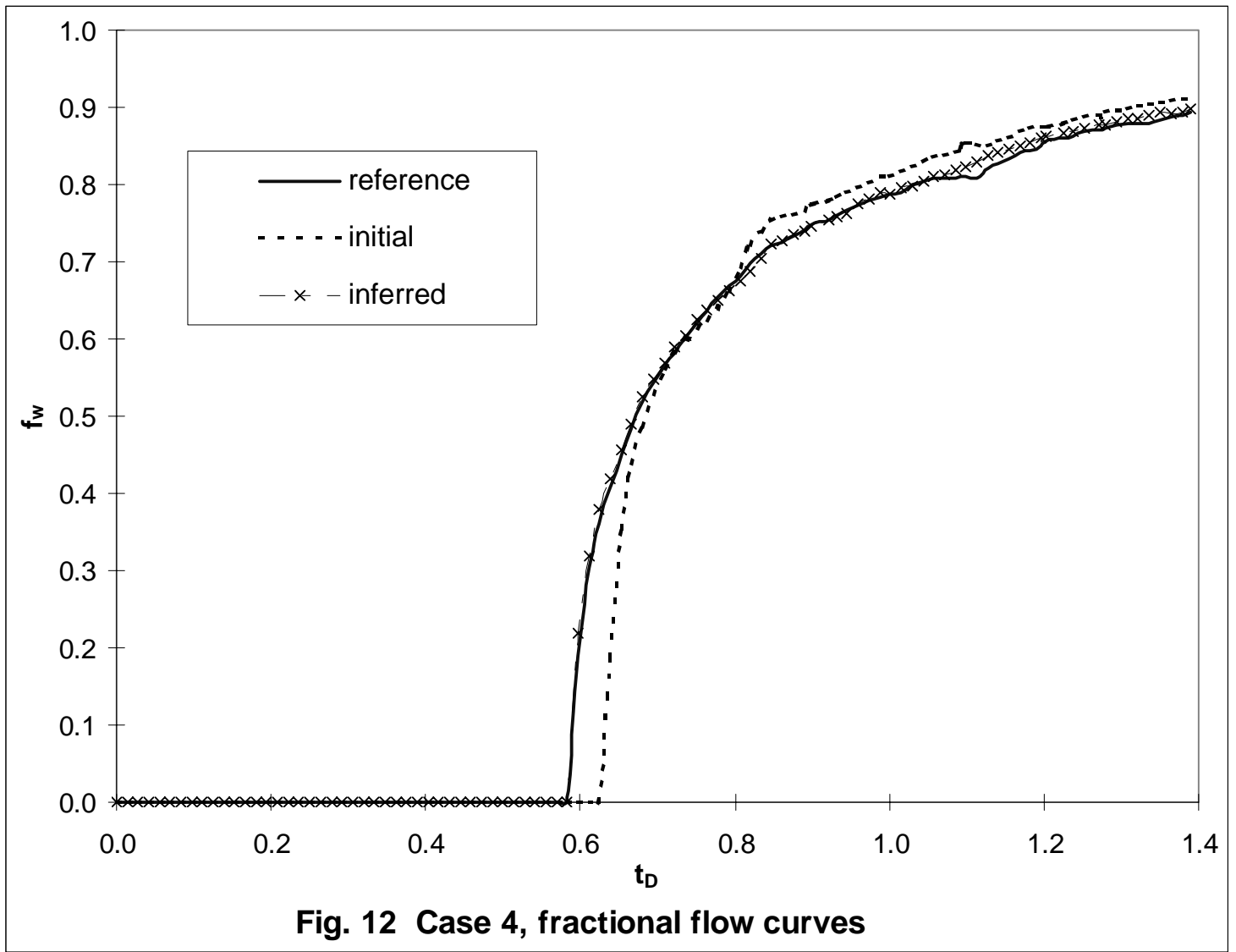




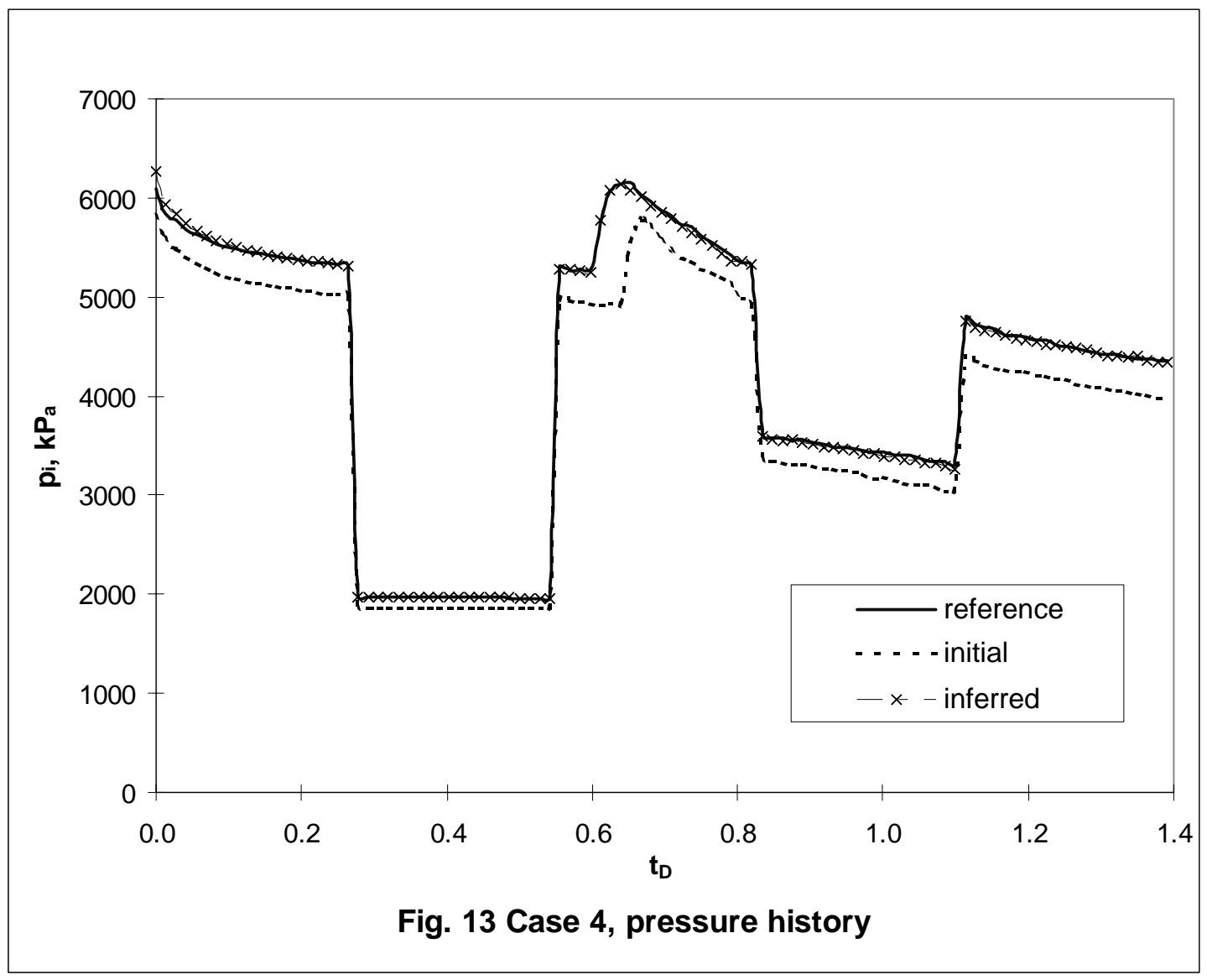




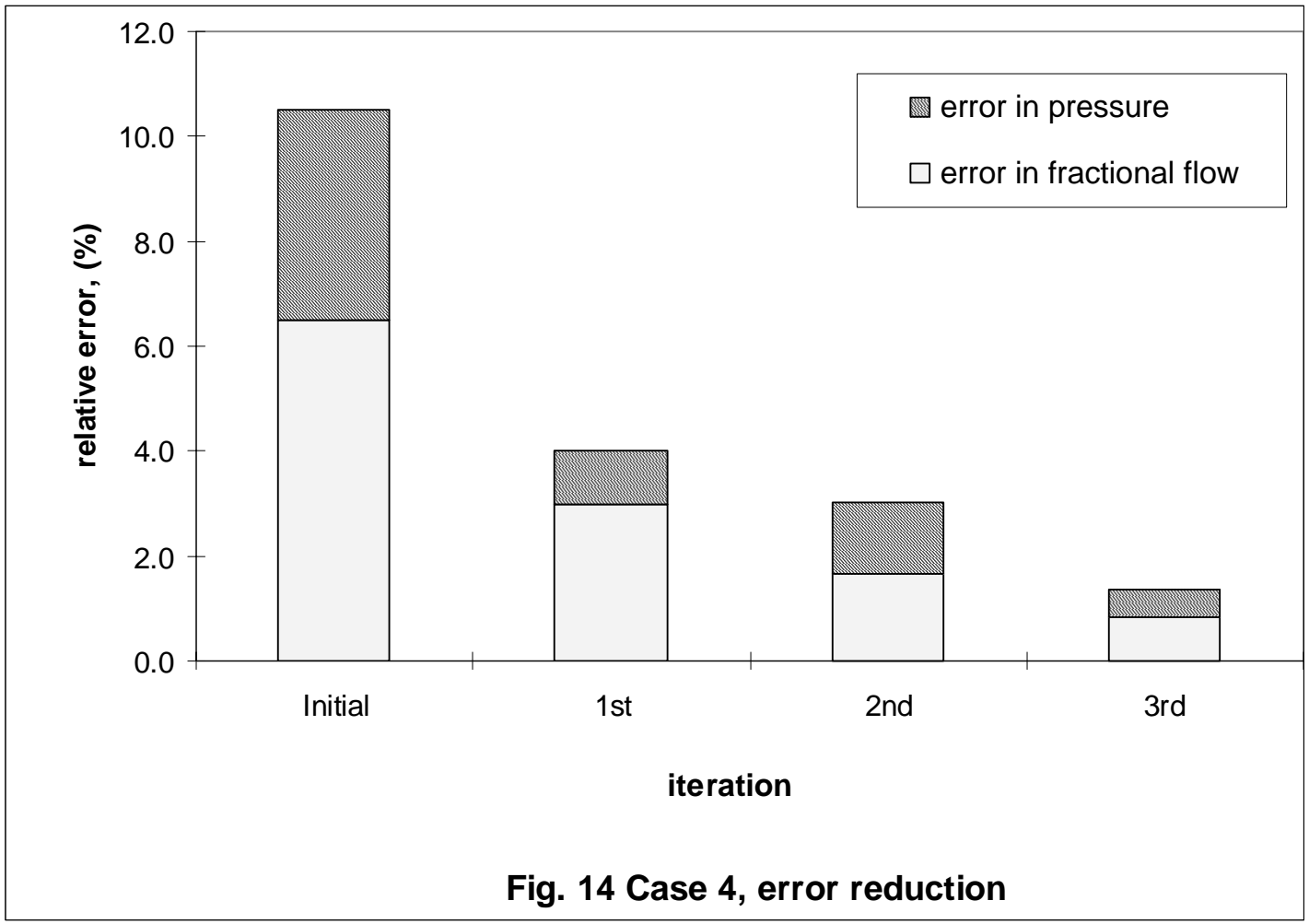

